\title{
A non-contact system for the assessment of hand motor tasks in people with Parkinson's disease
}

\author{
Fábio Henrique Monteiro Oliveira, 1 (D) Daniel Fernandes da Cunha ${ }^{3} \cdot$ Amanda Gomes Rabelo $^{2}$. \\ Luiza Maire David Luiz ${ }^{2} \cdot$ Marcus Fraga Vieira $^{4} \cdot$ Adriano Alves Pereira ${ }^{2} \cdot$ Adriano de Oliveira Andrade $^{2} \mathbb{D}$
}

Received: 17 May 2020 / Accepted: 21 December 2020 / Published online: 6 January 2021

(C) The Author(s) $2021 \quad$ OPEN

\begin{abstract}
Clinical diagnosis of Parkinson's disease (PD) motor symptoms remains a problem. Most of the current studies focus on objective evaluations to make the evaluation more reliable. Most of these systems are based on the use of inertial and electromyographic sensors that require contact with the body part being assessed. Contact sensors restrict natural movement, may be uncomfortable and may require preparation of the body, which may cause irritation. As an alternative to contact sensors for the study of hand motor tasks performed by subjects with and without PD, electrical potential sensing technology is used in this research. A custom hardware has been designed to enable data collection by hand movement. A micro-machine system validated the developed system, and a relationship model was established between hand displacement and non-contact capacitive (NCC) sensor response. An experiment was conducted, including 57 subjects, 30 with PD (experimental group) and 27 healthy control group, followed by an analysis of statistical features extracted from the instantaneous mean frequency (IMNF) of NCC sensor. These results were compared with those obtained from gyroscope signals that are considered in the field to be the gold standard. As a result, NCC responses were correlated linearly with hand displacement $\left(R^{2}=0.7692\right.$ and $\left.R_{a d j}^{2}=0.7631\right)$. The statistical evaluation of IMNF features showed, that both, contact and non-contact sensors, were able to discriminate movement patterns of the control group from the experimental one. The results confirm statistical similarity between features extracted from NCC and gyroscope signals.
\end{abstract}

Keywords Hand motor task · Instantaneous mean frequency · Non-contact capacitive sensor · Parkinson's disease

\section{Introduction}

Parkinson's disease (PD) is a neurodegenerative disease affecting about 6.1 millions of people worldwide [1]. Over the years, as a result of an increasing number of older people, the global burden of PD has become more critical [1, 2]. As worldwide life expectancy has increased it will likely continue to grow. The projected number of people with PD in the five most populous nations of Western Europe and in the ten most populous nations of the world for 2030 is 8.67 million, considering individuals over 50 years old [3].

PD is a very complex condition with several motor and non-motor symptoms that evolve over time for specialists

Supplementary Information The online version contains supplementary material available at https://doi.org/10.1007/s42452-020-04001 -5 .

Fábio Henrique Monteiro Oliveira, oliveirafhm@gmail.com; Daniel Fernandes da Cunha, danielcunha@ufg.br; Amanda Gomes Rabelo, amandagomesrabelo@gmail.com; Luiza Maire David Luiz, luizamaire@gmail.com; Marcus Fraga Vieira, marcus@ufg.br; Adriano Alves Pereira,

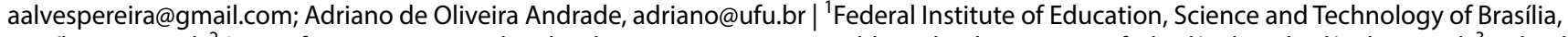
Brasília, DF, Brazil. ${ }^{2}$ Centre for Innovation and Technology Assessment in Health, Federal University of Uberlândia, Uberlândia, Brazil. ${ }^{3}$ Federal University of Goiás, Goiânia, Brazil. ${ }^{4}$ Bioengineering and Biomechanics Laboratory, Federal University of Goiás, Goiânia, Brazil. 
to treat optimally. Its diagnosis is purely clinical and relies on medical history and neurological evaluation [4]. Newly, the guidelines for clinical diagnosis of PD have been revised [5]. Nonetheless, the motor symptoms still reflect the traces of the disorder and are one of the most important features for PD diagnosis.

The Unified Parkinson's Disease Rating Scale (UPDRS) is a structured assessment tool that helps with disease progression and treatment response documentation [6]. The scale has four parts and measures complications of daily living non-motor experiences, daily living motor experiences, motor impairment, and complications of treatment. Such tools are still subjective despite the existence of scales to evaluate PD, and there is a strong dependence on the experience of the specialist.

These limitations in the diagnosis and follow-up of PD have motivated the development of new methods for PD assessment [7]. Wearable motion sensors, such as accelerometers, gyroscopes, magnetometers, and many other microelectronic devices have been used to obtain motor- and non-motor-related physiological information (i.e. anxiety, impaired decision-making, sleep disturbance) aspects of the disease [7]. These techniques are attempting to provide an objective way to measure the disease's motor symptoms with the intention of improving diagnosis, assessing disease progression, and evaluating the effectiveness of care and therapy.

Although several studies using these sensors show relevant results and a strong correlation with severity scales [7], the use of contact sensors presents challenges [8]. These sensors can cause skin irritation, require careful preparation of the skin and may not be used in situations with high risk of contact contamination [8,9]. On the other hand, the non-contact capacitive (NCC) sensor is an option for motion and biopotential analysis. This technology offers many advantages such as replicability, stability in long-term task monitoring, patient comfort and the possibility of application for daily monitoring [10].

There are some studies that introduced the use of NCC sensors in the context of motor quantification of PD. For example, the authors in [11] evaluated the motor tasks performed by a healthy person and one with PD. That paper describes the fundamental technology and provides some preliminary results. In a follow-up study [12], while healthy subjects were performing wrist movements, we compared the NCC sensor with gyroscope. The guiding hypothesis was that it is possible to quantify the main motor signs of Parkinson's disease, similar to one of the most commonly used inertial sensors, i.e., the gyroscope, by using the NCC electrical potential integrated circuit (EPIC) sensor.

In this study, it is presented the development of hardware and a wider application of this technology, based on

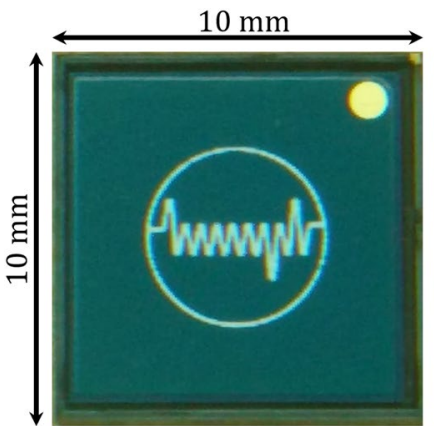

(a)

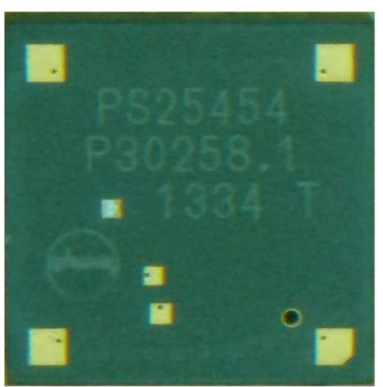

(b)
Fig. 1 PS25454 EPIC sensor (Plessey semiconductors, UK), a front and $\mathbf{b}$ rear views

the technology of electrical potential sensing (EPS) [13], for the evaluation of hand motor tasks performed by subjects with and without PD.

This research aims to address two issues: (1) modeling the relationship between NCC responses and displacement in a controlled scenario; (2) experimental evaluation of the implemented system by comparing results obtained from a gyroscope, whereas healthy individuals and people with PD performed different hand motor tasks.

The paper is structured as follows. Section 2 presents the materials and methods with the Sects. 2.1, 2.2, 2.3, 2.4, 2.5, 2.6 and 2.7. The results are presented in Sect. 3. In Sect. 4, the discussion about the 4.1 NCC system, 4.2 Waveforms and 4.3 Frequency analysis is presented. Lastly, conclusion and direction of future research are presented in Sect. 5.

\section{Materials and methods}

\subsection{Non-contact capacitive sensor}

The human body has dielectric properties and when it moves the electrical field is disturbed and NCC sensors are designed to detect this type of event [10]. By using this tool, different human tasks can be differentiated. Several studies have documented using this sensing technique for multiple purposes, including non-invasive respiration and cardiac monitoring [14], wearable devices to detect hair touch and restless leg movement [15] and passive tracking of movements [16]. However, we have not found a report on the use of capacitive sensors for the monitoring of hand movements in the context of PD.

The PS25454 EPIC capacitive sensor (see Fig. 1), used in our system, incorporates several features to enhance the measured signals, allowing the detection of field disturbance due to the movement of a nearby object. The main features of the employed sensor are ultra-high 


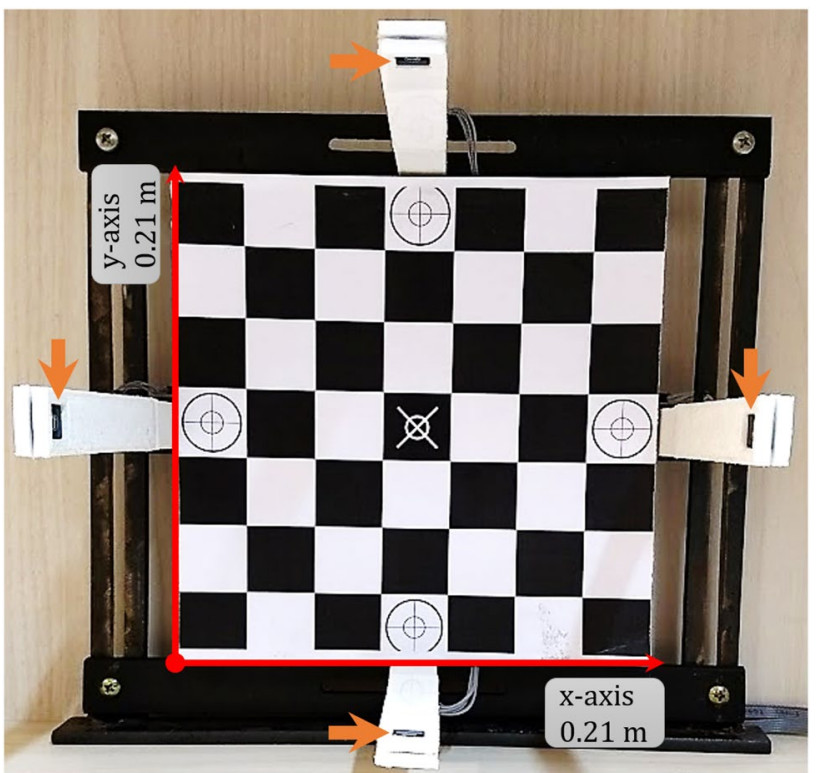

Fig. 2 2-D array of PS25454 sensors. Each pair of sensors provides information on movements in the $x$-axis and the $y$-axis. In the background, the marker board was specifically designed to assist the subject during the execution of experimental tasks. The orange arrow points to the four EPIC sensors

input resistance, typically $20 \mathrm{G} \Omega$; input capacitance as low as $15 \mathrm{pF}$; lower $-3 \mathrm{~dB}$ point typically $0.2 \mathrm{~Hz}$ and upper $-3 \mathrm{~dB}$ point typically $20 \mathrm{kHz}$. It works with bipolar power supply from $\pm 2.4 \mathrm{~V}$ to $\pm 5.5 \mathrm{~V}$.

This sensor (see Fig. 1) was designed with the specifications that made it suitable for the detection of movement in an open environment. When a person moves, the electrical field (E-field) is disturbed so that a relative signal can be used to estimate various position and motion-related information. One of the main advantages of this sensor technology is its ability to operate fully passively, without the need to emit waves in the environment, such as in the case of radar [17].

\subsection{System design}

Four PS25454 sensors were used in pairs in our system (National Institute of Intellectual Property-Brazil-BR 10 2018069864 8). The directional axis ( $x$ or $y$ ) is defined by each pair. A custom-made structure was designed using four sensors positioned at the four centered edges of a squared area of size $0.21 \mathrm{~m} \times 0.21 \mathrm{~m}$. Figure 2 shows this kind of arrangement.

The sensors were configured in differential mode to measure the local electrical field. In order to avoid common mode noise, INA333 (Texas Instruments, Dallas, TX, USA) instrumentation amplifier was used, which has high common mode rejection, low power consumption and low price.

The sensors require a power supply voltage of $\pm 5.5 \mathrm{~V}$, which is also appropriate for the instrumentation amplifier. In order to reduce environmental electrical noise, a battery was used as a power source.

The signals were fed to the instrumentation amplifier from both EPIC sensors. The output of the amplifier is then buffered by the voltage divider to adjust the signal level for analog-to-digital conversion (ADC). Figure 3 displays a simplified circuit diagram. The system was grounded to minimize interference with environmental noise.

\subsection{System validation}

A micro-machine unit (Minitech CNC Mini-Mill/GX, USA) with a resolution of $1 \mu \mathrm{m}$ was used as a guide for device validation. Attached to the reference system, the plaster hand model was fixed in order to simulate the use scenario. Figure 4 displays the experimental setup for the calibration of the device.

Two scenarios were considered in order to establish the relationship between the displacement and the voltage amplitude range. For the first, the hand was fixed $8 \mathrm{~cm}$ apart from the sensor indicated by a red asterisk (see Fig. 4)

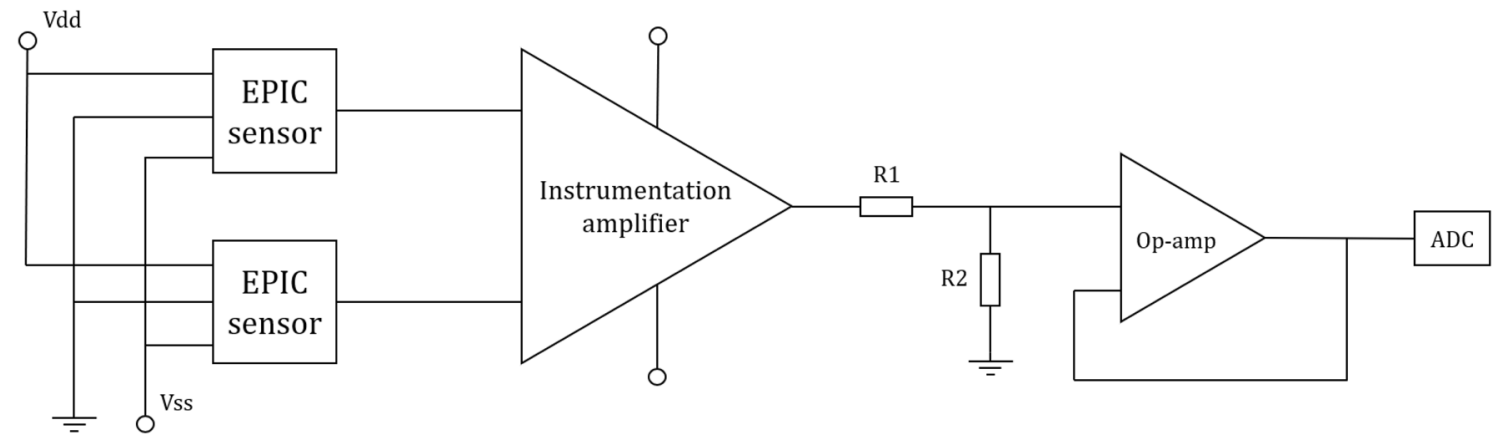

Fig. 3 Simplified circuit diagram to use PS25454 EPIC sensors in a differential mode for movement quantification 


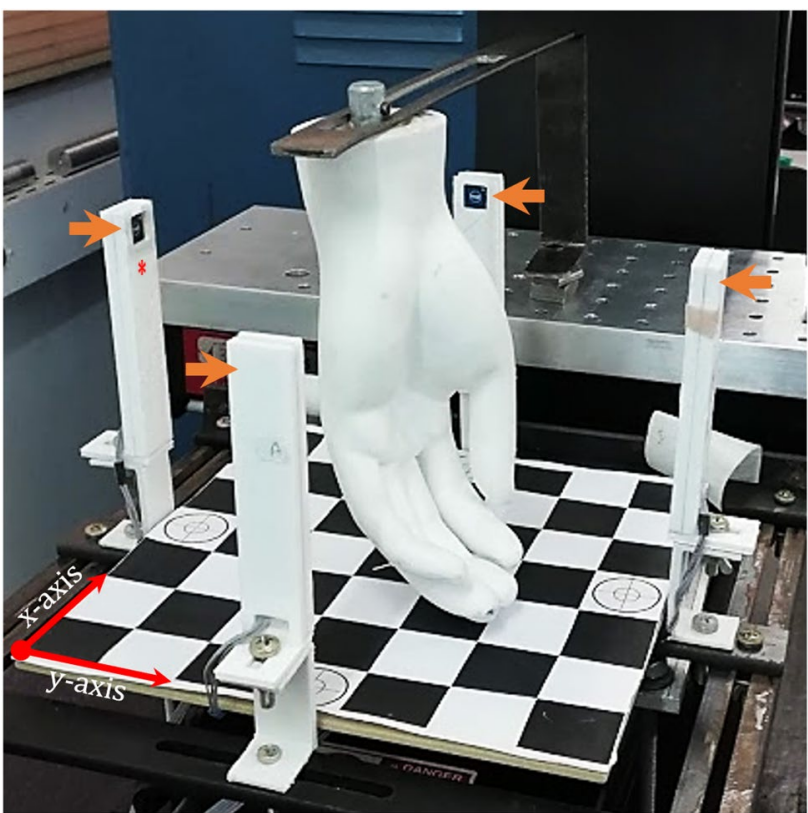

Fig. 4 Experimental configuration for testing of the NCC framework. The orientation of the axes is indicated by the red arrows and the orange arrows point to the four EPIC sensors. Each sensor is encapsulated in a customized framework

and for the second, the hand was fixed $4 \mathrm{~cm}$ apart from the same sensor. In both scenarios, the movements were along the $y$-axis and were executed three times each in the following sequence: (1) move $10 \mathrm{~mm}$; (2) move $5 \mathrm{~mm}$; (3) move $2.5 \mathrm{~mm}$; (4) move $1.5 \mathrm{~mm}$ and (5) move $0.5 \mathrm{~mm}$; always going back and forth.

The aim of this stage of the analysis was to establish the relationship between hand displacement and the response of the EPIC sensor. To do this, a linear mixed model [18] was used to describe the relationship between the variables measured,

$y \sim x+(1 \mid$ setup $)+\varepsilon$

where $x$ is our fixed effect term related to the hand displacement, $1 \mid$ setup is the random effect to model the two possible scenarios (i.e., the hand fixed at $4 \mathrm{~cm}$ and $8 \mathrm{~cm}$ apart from the reference sensor), and $\varepsilon$ is the general error term.

\subsection{Participants}

A total of 57 subjects were recruited, being 30 with $P D\left(S_{P D}\right)$ and 27 controls without $\mathrm{PD}\left(\mathrm{S}_{\mathrm{C}}\right)$. All the subjects signed a written informed consent, and the study procedure was approved by the National Research Ethics Committee (65165416.4.0000.5152). Each group of subjects was paired in terms of age (equal medians with $p=0.56$ ) and
Table 1 Clinical data of subjects with PD $\left(\mathrm{S}_{\mathrm{PD}}\right)$

\begin{tabular}{|c|c|c|c|c|}
\hline Subject & Age & Sex & $\begin{array}{l}\text { UPDRS Part III } \\
\text { score* }\end{array}$ & $\begin{array}{l}\text { Hoehn and } \\
\text { Yahr stage* }\end{array}$ \\
\hline 1 & 63 & M & 34 & 2 \\
\hline 2 & 54 & $M$ & 30.7 & 2 \\
\hline 3 & 47 & $M$ & 31.7 & 2 \\
\hline 4 & 76 & $M$ & 30.3 & 2 \\
\hline 5 & 74 & M & 15.7 & 1.3 \\
\hline 6 & 70 & M & 29.5 & 2 \\
\hline 7 & 48 & M & 45.7 & 2 \\
\hline 8 & 56 & $\mathrm{~F}$ & 17.3 & 1.7 \\
\hline 9 & 48 & $\mathrm{~F}$ & 43.3 & 2 \\
\hline 10 & 60 & M & 62.3 & 2 \\
\hline 11 & 64 & M & 43.3 & 2 \\
\hline 12 & 55 & $\mathrm{~F}$ & 24.3 & 2 \\
\hline 13 & 69 & M & 56.3 & 3 \\
\hline 14 & 53 & $\mathrm{~F}$ & 20 & 1.7 \\
\hline 15 & 50 & $\mathrm{~F}$ & 30.5 & 2.5 \\
\hline 16 & 73 & M & 49.7 & 1.3 \\
\hline 17 & 72 & $\mathrm{~F}$ & 27 & 2 \\
\hline 18 & 67 & $M$ & 14.7 & 1.7 \\
\hline 19 & 62 & $M$ & 52 & 3 \\
\hline 20 & 66 & M & 43 & 2 \\
\hline 21 & 62 & $\mathrm{~F}$ & 10 & 1 \\
\hline 22 & 78 & $M$ & 45 & 2 \\
\hline 23 & 56 & $M$ & 38 & 2 \\
\hline 24 & 61 & $\mathrm{~F}$ & 34.7 & 2 \\
\hline 25 & 49 & M & - & - \\
\hline 26 & 62 & $\mathrm{~F}$ & 33 & 2 \\
\hline 27 & 60 & $\mathrm{~F}$ & 34 & 3 \\
\hline 28 & 72 & M & 37 & 2.5 \\
\hline 29 & 55 & M & 20 & 2 \\
\hline 30 & 82 & M & 15 & 1 \\
\hline Summary & $62.1 \pm 9.7$ & $20 \mathrm{M}, 10 \mathrm{~F}$ & $33.4 \pm 13.3$ & $2 \pm 0.1$ \\
\hline
\end{tabular}

* Mean of three qualified evaluators

sex. Subjects with PD were evaluated using MDS-UPDRS by three qualified evaluators and the disease progression to different Hoehn and Yahr (HY) stages was also evaluated by three qualified evaluators. Table 1 shows characteristics of the subjects with PD. The information summary of controls group $\left(\mathrm{S}_{C}\right)$ are: age $=60.7 \pm 8.5$ and $\mathrm{sex}=17 \mathrm{M}, 10 \mathrm{~F}$. The results of UPDRS and HY scores provided in the table are the overall mean between evaluators.

MDS-UPDRS Part III concerns the motor test with a total score ranging from 0 to 132 [6]. The overall mean score of Part III provided in Table 1 reflects the PD severity of our sample from the $S_{P D}$ group. As a figure, this is $25 \% \pm 9 \%$. In clinical terms, this score is correlated with a moderate to mild PD motor test severity. "Moderate" refers to signs with sufficiently low frequency or intensity to cause no impact 


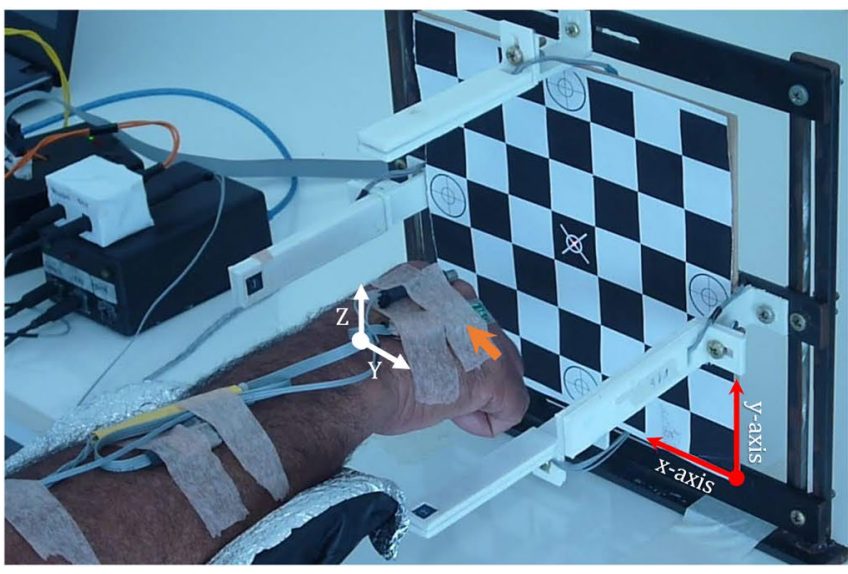

Fig. 5 The experimental setup. (left) The 3-axial gyroscope is positioned along with the laser point on the dorsal part of the hand. The orange arrow is pointing to the gyroscope sensor. The $y$-axes and $z$-axes are marked. The forearm is on the support. During the

on function and "mild" refers to signs of frequency or intensity sufficient to cause a modest impact on function [6].

\subsection{Experimental setup}

The experiments ${ }^{1}$ were performed in an open unshielded environment (Fig. 5). A three-axial gyroscope (L3GD2OH, STMicroelectronics, Switzerland) was placed on the dorsal region of the hand along with a laser pointer to assist the subject in performing a set of motor tasks (Fig. 5). The TREMSEN (Precise Tremor Sensing Technology) system (National Institute of Intellectual Property—Brazil—BR 10 20140232826 ) was used for data acquisition and real-time visualization of the signals [19].

Subjects were instructed to start a motor task with a visual laser point in the central region of the board (highlighted by a crossed circle). The white circles on the board were set as targets for each type of experimental task (Fig. 5). For task T1, subjects were instructed to hold a hand in a position against gravity with a visual laser point in the central region of the board for $10 \mathrm{~s}$. For tasks T2 to T5, subjects were asked to perform hand movements 5 times in $10 \mathrm{~s}$, while for tasks T6 to T9, subjects were asked to perform the same type of tasks 15 times in $10 \mathrm{~s}$. This sequence was performed three times with each execution having a rest.

While the selected motor tasks are not in the UPDRS, they are closely related to those performed by patients during the UPDRS evaluation [6]. Gyroscopes are also suitable for evaluating the selected movements as they detect angular velocity, which may vary depending on the task.

1 A video of an experiment trial of a subject with PD was uploaded, and it is available under section of the Supplementary Files.

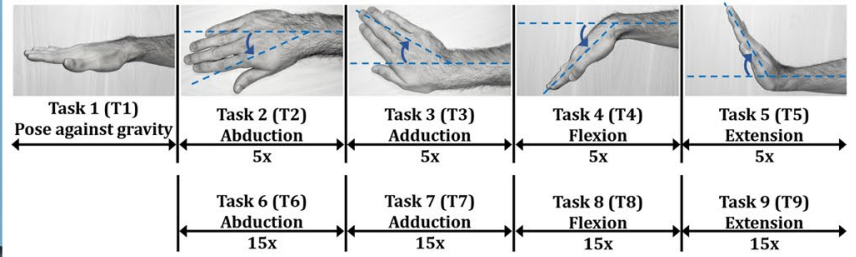

experiment, the squared board is used to guide the participant. (right) Set of tasks performed. The number of repetitions is shown for each task

Signals collected from the gyroscope were sampled at $50 \mathrm{~Hz}$ (with sensitivity of $\pm 500 \%$ s) [20] and from the NCC system at $3 \mathrm{kHz}$. This sample rate was chosen because of the sensor behavior, in a way that, after carrier removal, 50 points are left for every second. The signals were synchronized by an external trigger. The $x$ and $y$ axes of the NCC system correspond to the $z$ and $y$ coordinates of the gyroscope, respectively.

\subsection{Signal pre-processing}

The NCC sensor signal comes with an amplitude modulation carrier at $60 \mathrm{~Hz}$ (power line frequency). Thus, the carrier was removed using an envelope technique to extract the relevant information from the output sensor signal. The mains frequency $(50 \mathrm{~Hz} / 60 \mathrm{~Hz})$ can be measured by each sensor. When a dielectric object, such as a hand, is positioned among two sensors, the level of the main signal detected by the sensor is altered.

The signals collected from the gyroscope were pre-processed by using a moving average filter and a nonlinear detrending technique described in [21]. For the signals detected from the NCC system, the sequence of steps was employed, first the signal envelope of the peaks at $60 \mathrm{~Hz}$ component was estimated to remove the carrier signal, followed by the same sequence of steps applied to the gyroscope.

\subsection{Frequency analysis}

The NCC sensor detects disturbances in the local E-field and translates this into a signal consisting of the sum of all disturbances detected in the E-field. The following steps were applied to the signals from both sensors 


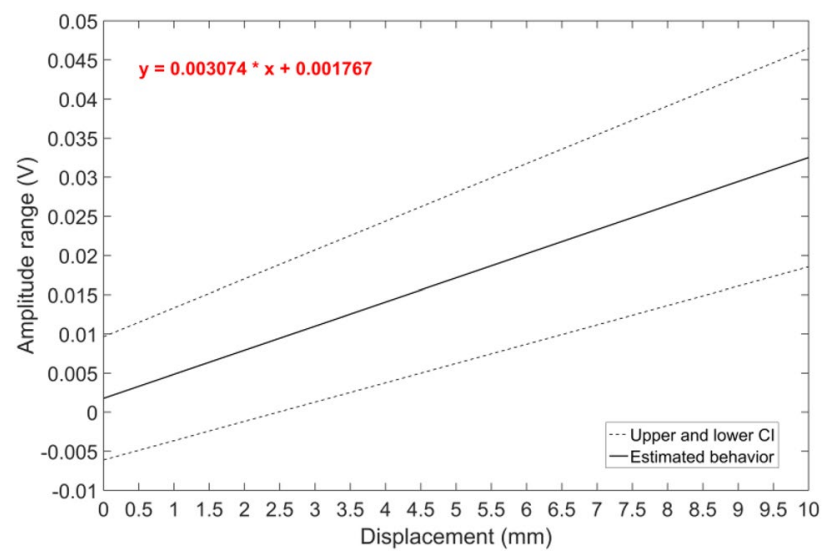

Fig. 6 Relationship between displacement and amplitude range of NCC EPIC sensor. The dashed lines represent upper and lower $95 \%$ confidence intervals. In red on the upper left corner is the line equation of the linear mixed model

(i.e. NCC and gyroscope): (1) estimate the instantaneous mean frequency (IMNF) based on Hilbert spectrum (HS) [22]; (2) calculate some of the key values of the descriptive statistics (i.e., min-minimum value excluding outliers, q1 - first quartile, median, q3 -third quartile and max-maximum value excluding outliers). Points are treated as outliers if they are greater than $q 3+w \times(q 3-q 1)$ or less than $q 1-w \times(q 3-q 1)$, where $w$ is the maximum whisker length, and $q 1$ and $q 3$ are the 25 th and 75 th percentiles of the sample data, respectively; (3) apply one-sample Kolmogorov-Smirnov test to check the normality of the distribution; (4) apply a statistical test to check the statistical significance of the differences between the $S_{P D}$ and $S_{C}$ groups, considering the estimated descriptive statistical features for each axis.

\section{Results}

\subsection{System validation}

As a result of the experiment described in Sect. 2.3, Fig. 6 shows the model response of NCC EPIC sensor.

As shown in Fig. 6, the model presented strong linearity. R-squared and adjusted R-squared were nearly the same $\left(R^{2}=0.7692\right.$ and $\left.R_{a d j}^{2}=0.7631\right)$.

The following linear equation

$y=0.003074 x+0.001767$

was estimated by means of the fitted model, where $x$ is the displacement in millimeters and $y$ the sensor output value in volts. From (2) it is possible to estimate the sensor behavior.

\subsection{Typical waveforms}

Figure 7 illustrates characteristic waveforms obtained for a subject from $\mathrm{S}_{\mathrm{C}}$ and $\mathrm{S}_{\mathrm{PD}}$ groups while executing the tasks shown in Fig. 5.

In Fig. 7, the signals from both sensors can be visually compared and a high similarity between their waveform can be seen. Each type of movement occurs most intensely on a specific axis. For instance, T4 and T5, i.e., flexion and extension, respectively, occur mostly on the $y$-axis. Nevertheless, it is also possible to observe smaller scale contribution on the $z$-axis to the gyroscope sensor, and on the $x$-axis to the NCC sensor, latter if the signal is zoomed in.

\subsection{Frequency analysis}

The estimation of IMNF for frequency analysis was made by means of the $\mathrm{HS}$, for all subjects considering three trials and the nine tasks. Figure 8 shows an example of the estimation of IMNF, for signals collected from the task 2-abduction.

The differences between signal waveforms for $S_{C}$ and $S_{P D}$ subjects can be seen in Fig. 8 . In the first $2 \mathrm{~s}$ of Fig. $8 \mathrm{~b}$, it is possible to see oscillations in the signals of gyroscope and NCC sensor, latter to a lesser extent. These oscillations come from hand shaking, and they are also present throughout the signal, similar to transients.

Table 2 presents a comparison between $S_{C}$ and $S_{P D}$ groups considering the estimated IMNF values for both sensors (i.e., NCC and gyroscope) and for each task. The $p$-values highlighted in bold help in visualization of which sensor axes and features not reached a significance level of $95 \%(p<0.05)$.

In most cases, both sensors were able to discriminate features from signals of each group $\left(\mathrm{S}_{\mathrm{C}}\right.$ and $\left.\mathrm{S}_{\mathrm{PD}}\right)$. For $\mathrm{NCC}$ sensor, tasks 1 and 3 were not discriminated completely by considering both axes. The gyroscope sensor, in turn, did not show statistical significance for tasks $1,3,4,5,6,8$, and 9 .

\section{Discussion}

This research proposed the use of non-contact capacitive sensors for the assessment of people with Parkinson's disease. The results showed that the NCC sensor acts as well as a gyroscope sensor for discrimination against movements performed by people with and without PD.

The fact that PD has yet to be cured challenges science, and this is not the only challenge. Diagnosis and follow-up of PD is another, and over the years several studies have focused on the detection of motor and non-motor PD symptoms. PD was originally considered a pure motion 
Fig. 7 Typical signals collected. a subject without PD, b subject with PD. Each rectangular window highlighted in red represents the signal from a task

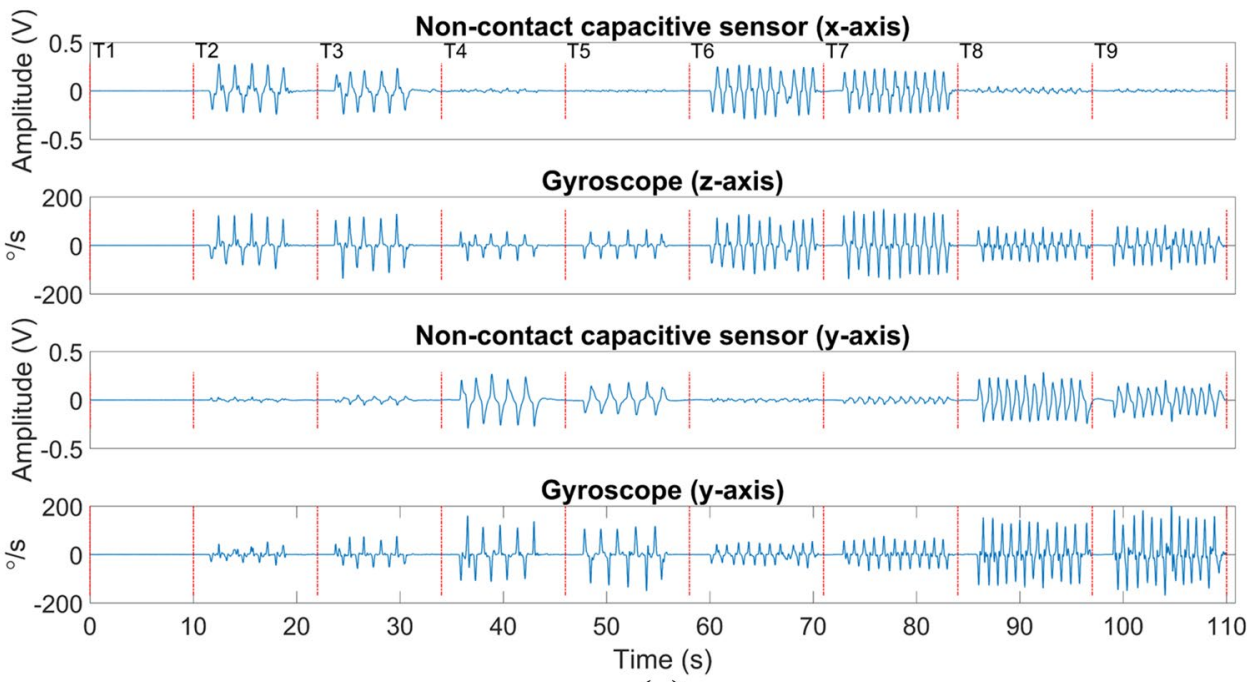

(a)
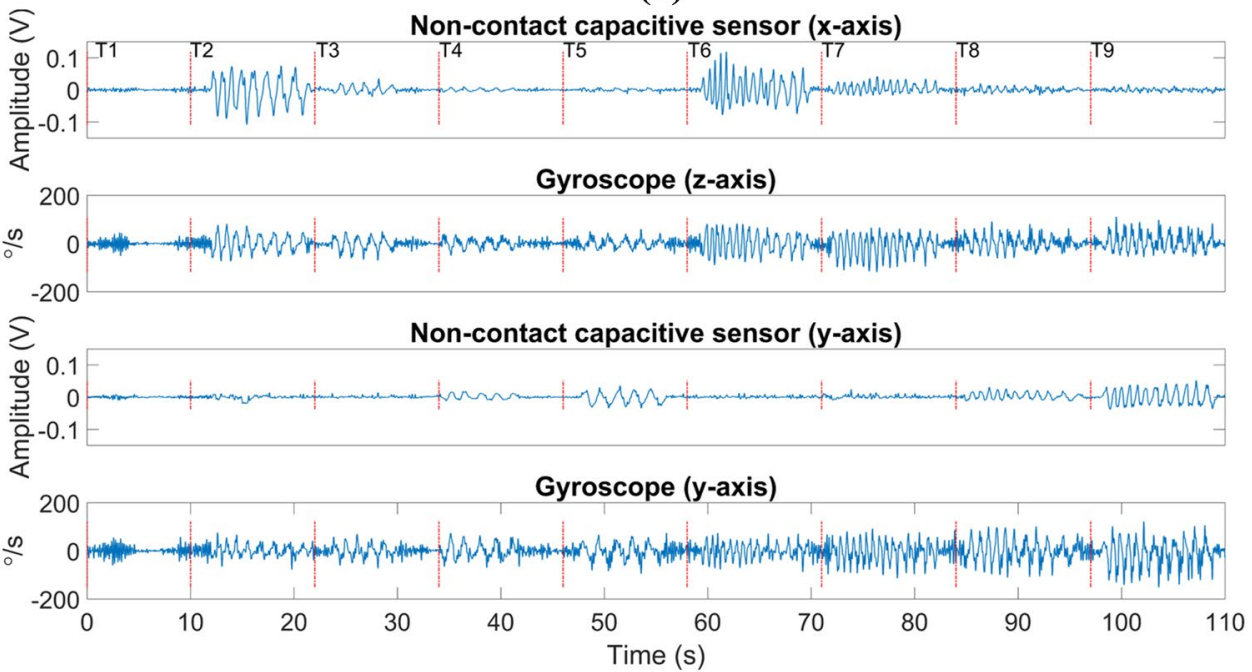

(b) disorder characterized by its main motor symptoms, also known as cardinal signs, i.e. bradykinesia, stiffness, tremor and postural instability [23] and these motor signs worsen over time.

Therefore, many sensors and techniques are employed to quantify the cardinal signs of PD. Most studies [7, 24, 25] address this challenge by using contact sensors (e.g., inertial sensors), and this method has limitations. As a result, the alternative use of NCC sensors offers new possibilities, in addition to overcoming certain drawbacks related to the contact approach.

The analysis of the tasks depicted in Fig. 5 can support the development of a score to quantify the severity of some of cardinal signs. For example, Amanda et al. [20] studied bradykinesia by quantifying the flexion and extension of the wrist. Pose versus Gravity was evaluated in [26] for the estimation of hand tremor parameters.
In addition, NCC systems can be helpful in many ways: (1) for people with sensitive skin, such as the elderly, the majority of whom suffer from PD [8]; (2) for invisible application, once they work without contact and even through the wall [27]; (3) as a tool to allow examination in environments with high risk of contamination by contact [9]; (4) less interference due to cable movements; (5) privacy concerns and (6) less amount of data and processing time while compared with image-based systems.

\subsection{NCC system}

To date, there have been only a few studies using EPIC sensors, especially non-contact versions. The data sheet of these sensors does not contain enough information, and the lack of application notes makes its use difficult.

González-Sánchez et al. [14] used EPIC sensors to develop an electrocardiographic (ECG) monitoring system 
Fig. 8 Typical IMNFs (in green), estimated through the HS, for task 2. a Signals and IMNFs for a subject without PD, for the $\mathrm{x}$-axis of both sensors. $\mathbf{b}$ Signals and IMNFs for a subject with PD, for the $x$-axis of both sensors. Selected subjects are matched in age and sex
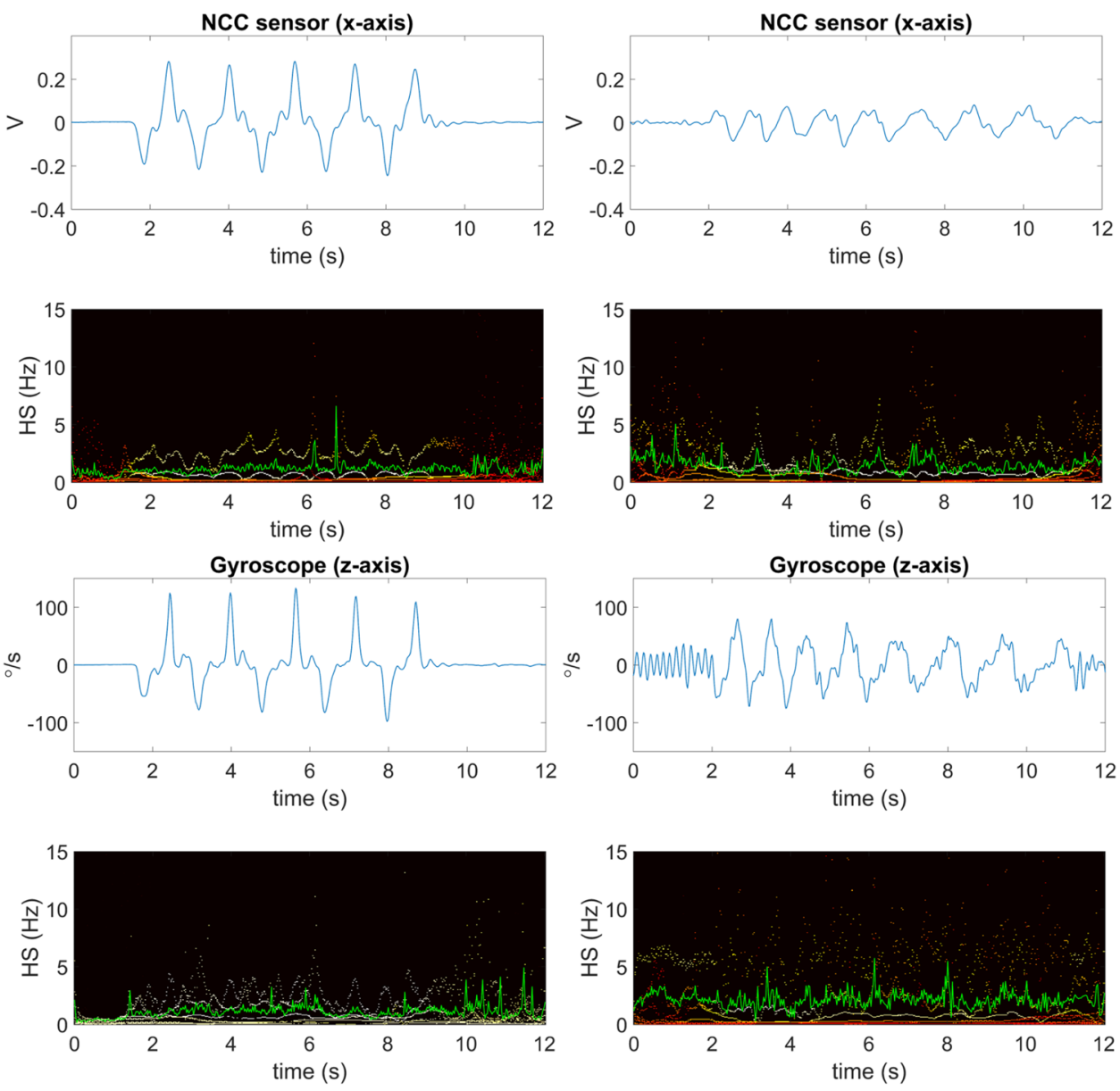

(a)

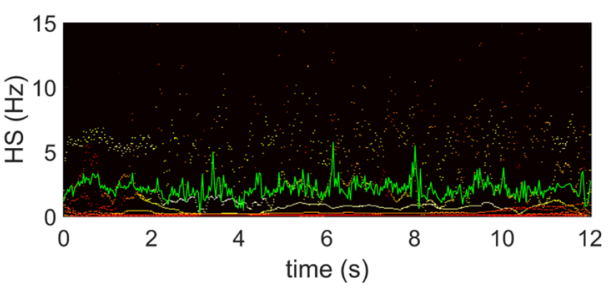

(b) that does not require skin preparation or direct contact. From a sensor instrumentation circuit point of view, their study presented very well the circuit design and some faced issues. The PS25251 EPIC sensor was used in their research and, according to its datasheet, this variant of the sensor was designed to be used as an ECG dry contact sensor. However, their first test setup of two differential sensors was used in a contactless approach. A laboratory mouse was placed between the sensors in this setup. In the end, a plastic tube was used with ground aluminum foil to reduce the electrical noise detected by the sensors.

Two approaches have been tested in our study. In the first, the NCC system was designed to operate in a singleended mode. As a result, each sensor individually captured the E-field disturbance in such a way that the amplitude of the signal corresponding to the sensor unit increases when the subject's hand approaches it. After that, in order to determine each axis, the signals, after digitization, were subtracted from each pair of sensors. This approach was presented as not stable and the signals were often swamped by environmental noise.
A differential mode between a pair of sensors was adopted in the second approach. Most of the time, using this method, the signal of interest was not swamped by environmental noise. After digitization, the $60 \mathrm{~Hz}$ signal appeared as a carrier, and the signal of interest varied the amplitude of the carrier, as did the amplitude modulation (AM). However, even using differential mode design, the signal of interest (i.e., voluntary and involuntary movements of the hand) sometimes seemed weak and was heavily swamped by environmental noise. In order to overcome this limitation, the grounding technique also referred to in [14] was used, i.e. the support of the arm was covered by aluminum foil connected to the ground (GND). Using this grounding technique, the signals were stable during all data collections.

As far as device validation is concerned, the calculated model shown in Fig. 6 provided an agreement with the measurements made by Aydin et al. [28]. The differences in amplitude range between our results and those presented in [28] are due to measured phenomena. In our experiment, the electrical field disturbance due to hand displacement was measured and, in their experiment, 
Table 2 Comparison of descriptive statistics based on IMNF values between $\mathrm{S}_{\mathrm{C}}$ and $\mathrm{S}_{\mathrm{PD}}$ groups by Mann-Whitney U-test

\begin{tabular}{|c|c|c|c|c|c|}
\hline & & \multicolumn{2}{|c|}{$\begin{array}{l}\text { NCC sensor } \\
p \text { value }\end{array}$} & \multicolumn{2}{|c|}{$\begin{array}{l}\text { Gyro sensor } \\
p \text {-value }\end{array}$} \\
\hline & & $x$-axis & $y$-axis & x-axis & $y$-axis \\
\hline \multirow[t]{5}{*}{ Task 1} & $\min$ & 0.00 & 0.06 & 0.02 & 0.00 \\
\hline & $q 1$ & 0.00 & 0.54 & 0.28 & 0.08 \\
\hline & median & 0.00 & 0.87 & 0.01 & 0.00 \\
\hline & q3 & 0.00 & 0.92 & 0.00 & 0.00 \\
\hline & $\max$ & 0.02 & 0.82 & 0.00 & 0.00 \\
\hline \multirow[t]{5}{*}{ Task 2} & $\min$ & 0.00 & 0.00 & 0.00 & 0.00 \\
\hline & q1 & 0.00 & 0.00 & 0.00 & 0.00 \\
\hline & median & 0.00 & 0.00 & 0.00 & 0.00 \\
\hline & $q^{3}$ & 0.00 & 0.00 & 0.00 & 0.00 \\
\hline & $\max$ & 0.00 & 0.00 & 0.00 & 0.01 \\
\hline \multirow[t]{5}{*}{ Task 3} & $\min$ & 0.00 & 0.00 & 0.03 & 0.05 \\
\hline & q1 & 0.00 & 0.01 & 0.00 & 0.00 \\
\hline & median & 0.00 & 0.01 & 0.00 & 0.00 \\
\hline & q3 & 0.00 & 0.03 & 0.00 & 0.00 \\
\hline & $\max$ & 0.00 & 0.06 & 0.00 & 0.00 \\
\hline \multirow[t]{5}{*}{ Task 4} & $\min$ & 0.00 & 0.00 & 0.01 & 0.00 \\
\hline & $q 1$ & 0.00 & 0.00 & 0.00 & 0.00 \\
\hline & median & 0.00 & 0.00 & 0.00 & 0.00 \\
\hline & q3 & 0.00 & 0.00 & 0.01 & 0.00 \\
\hline & $\max$ & 0.00 & 0.00 & 0.05 & 0.00 \\
\hline \multirow[t]{5}{*}{ Task 5} & $\min$ & 0.00 & 0.00 & 0.02 & 0.00 \\
\hline & $q 1$ & 0.00 & 0.00 & 0.00 & 0.00 \\
\hline & median & 0.00 & 0.00 & 0.00 & 0.00 \\
\hline & $q^{3}$ & 0.00 & 0.00 & 0.02 & 0.00 \\
\hline & $\max$ & 0.00 & 0.00 & 0.11 & 0.00 \\
\hline \multirow[t]{5}{*}{ Task 6} & $\min$ & 0.00 & 0.00 & 0.00 & 0.00 \\
\hline & q1 & 0.00 & 0.00 & 0.00 & 0.02 \\
\hline & median & 0.00 & 0.00 & 0.00 & 0.03 \\
\hline & q3 & 0.00 & 0.00 & 0.00 & 0.10 \\
\hline & $\max$ & 0.00 & 0.00 & 0.00 & 0.36 \\
\hline \multirow[t]{5}{*}{ Task 7} & $\min$ & 0.00 & 0.00 & 0.00 & 0.01 \\
\hline & q1 & 0.00 & 0.00 & 0.00 & 0.00 \\
\hline & median & 0.00 & 0.00 & 0.00 & 0.00 \\
\hline & $q^{3}$ & 0.00 & 0.00 & 0.00 & 0.00 \\
\hline & $\max$ & 0.00 & 0.00 & 0.00 & 0.00 \\
\hline \multirow[t]{5}{*}{ Task 8} & $\min$ & 0.00 & 0.00 & 0.13 & 0.02 \\
\hline & q1 & 0.00 & 0.00 & 0.05 & 0.00 \\
\hline & median & 0.00 & 0.00 & 0.03 & 0.00 \\
\hline & q3 & 0.00 & 0.00 & 0.02 & 0.00 \\
\hline & $\max$ & 0.00 & 0.00 & 0.02 & 0.00 \\
\hline \multirow[t]{5}{*}{ Task 9} & $\min$ & 0.00 & 0.00 & 0.08 & 0.04 \\
\hline & $\mathrm{q} 1$ & 0.00 & 0.00 & 0.04 & 0.05 \\
\hline & median & 0.00 & 0.00 & 0.01 & 0.03 \\
\hline & q3 & 0.00 & 0.00 & 0.00 & 0.01 \\
\hline & $\max$ & 0.00 & 0.00 & 0.00 & 0.00 \\
\hline
\end{tabular}

the goal was to calibrate the EPIC sensor as a field meter, thus establishing a known electrical field in the research laboratory.

Although the variations between the movements performed in the device validation (i.e. hand translation) and the experimental process (i.e. hand rotation), the basic principle of the NCC EPIC sensor was modeled and illustrated in Fig. 7. This phase was important to confirm the previous result [28] and to move forward. This kind of device testing could be expanded, considering, for example, a number of hand movements.

\subsection{Waveforms}

The results obtained suggest that both sensors, the NCC and the gyroscope, can measure all assessed movements (i.e., T1 to T9). The signal shapes of the NCC and the gyroscope sensor are very similar to those shown in Fig. 7. For example, a correlation study was carried out in [12], and the results showed a high degree of agreement between the two sensors. This similar behavior between them is important once the gyroscope sensor is commonly used and well known in the field of motion analysis, including the PD motor symptom assessment [7].

On the other hand, the NCC system is highly dependent on the design and calibration of the sensors. This problem can be seen in Fig. 7b, where the signal amplitude of the $\mathrm{NCC}$ sensor for the $\mathrm{x}$-axis is higher than for the $y$-axis. There are at least two possible reasons for this phenomenon: (1) the subject's hand is closer to the sensors of $x$-axis, once the human hand is wider in transverse plane than in the sagittal plane; (2) in Fig. 7b, the subject has PD and he suffers from bradykinesia (score 3 for item 3.4 of MDS-UPDRS) and rigidity (score 2 for item 3.3 of MDS-UPDRS), which usually leads to slow movement and decreased motion range. These two symptoms directly affect the movements of the subject's hand that are reflected in the NCC sensor signal captured. The latter reason can also be noted, but to a lesser degree, in the gyroscope sensor signal.

\subsection{Frequency analysis}

Frequency domain information is important and commonly used for evaluating PD motor symptoms [24, 25, 29].

In Fig. 8, for example, IMNFs have been estimated over time. Figure $8 \mathrm{~b}$ shows the task signal and its IMNF for the PD subject. Tremor symptom appears while analyzing signals from both sensors, and the frequency estimation method used was able to track signal frequency oscillation. This phenomenon can be visually observed in the 
first $2 \mathrm{~s}$ of Fig. $8 \mathrm{~b}$ for both sensors where the subject had a severe hand tremor.

A comparison of descriptive statistics based on IMNF values between $\mathrm{S}_{\mathrm{C}}$ and $\mathrm{S}_{\mathrm{PD}}$ groups is shown in Table 2. Both sensors distinguish very well the motor patterns of both groups. Overall, the NCC sensor was performing better. In many cases where the NCC or gyroscope did not have statistical significance for a particular axis, the possible reason is that the movement analyzed occurred mainly on the other axis. For example, task 3 occurs primarily on the $x$-axis. The result of the NCC sensor in task 1 on the $y$-axis should be highlighted. In task 1, the subject was asked to maintain a posture against gravity, and in this posture, even if the subject's hand shakes, it would be closer to the $x$-axis sensors and proportionally away from the $y$-axis sensors, given the anatomical dimensions of the hand.

\section{Conclusion and future work}

This paper described a new application of the non-contact sensing technology, which is completely passive and is working to detect variations in the electrical field of the ambient. We have shown that, with the custommade arrangement of electrical potential sensors, hand movements can be quantified. The system was calibrated and applied for the assessment of wrist motor tasks performed by subjects with and without Parkinson's disease. This technology and the application presented in this paper open up new possibilities for the field of movement analysis.

A comparison was made using the estimated frequency features of the NCC and gyroscope signals, together with a statistical significance check. In view of this scenario, the NCC system has been shown to be as successful as the gyroscope-based system.

In addition to the practicality of using the proposed system on a daily basis, it is also suitable for people with skin problems, such as sensitive skin in the case of the elderly. It could be used in environments with a high risk of contact contamination. While compared to image-based systems, there are no privacy concerns once the proposed system does not require the capture and/or storage of data to be processed.

In addition, other experimental configurations could be evaluated in future studies, as the literature shows that distance and number of sensors impact directly on the acquired signal $[14,28]$. Studies to turn this system into wearable could also be relevant to the field.

Acknowledgement The authors are thankful to the volunteers and to the patients of the Parkinson's disease Association of Triângulo
(Associação de Parkinson do Triângulo, Uberlândia, Brazil) for their participation in this study.

Funding The present work was carried out with the support of the National Council for Scientific and Technological Development (CNPq), Coordination for the Improvement of Higher Education Personnel (CAPES - Program CAPES/DFATD-88887.159028/2017-00 and Program CAPES/COFECUB-88881.370894/2019-01), the Foundation for Research Support of the State of Minas Gerais (FAPEMIGAPQ-00942-17) and Foundation for Research Support of the Federal District (FAPDF). A. O. Andrade is fellow of CNPq, Brazil (304818/20186). M. F. Vieira is fellow of CNPq, Brazil (306205/2017-3). A. A. Pereira is fellow of CNPq, Brazil (310911/2017-6).

\section{Compliance with ethical standards}

Conflict of interest The authors have no conflict of interest to disclose. In addition, Fábio Henrique Monteiro Oliveira and Adriano de Oliveira Andrade have a patent BR 1020180698648 pending.

Ethics approval This study procedure was approved by the National Research Ethics Committee (CAAE: 65165416.4.0000.5152 23/02/2017)

Informed consent Informed consent was obtained from all individual participants included in the study.

Open Access This article is licensed under a Creative Commons Attribution 4.0 International License, which permits use, sharing, adaptation, distribution and reproduction in any medium or format, as long as you give appropriate credit to the original author(s) and the source, provide a link to the Creative Commons licence, and indicate if changes were made. The images or other third party material in this article are included in the article's Creative Commons licence, unless indicated otherwise in a credit line to the material. If material is not included in the article's Creative Commons licence and your intended use is not permitted by statutory regulation or exceeds the permitted use, you will need to obtain permission directly from the copyright holder. To view a copy of this licence, visit http://creativecommons .org/licenses/by/4.0/.

\section{References}

1. Dorsey ER, Elbaz A, Nichols E et al (2018) Global, regional, and national burden of Parkinson's disease, 1990-2016: a systematic analysis for the global burden of disease study 2016. Lancet Neurol 17:939-953. https://doi.org/10.1016/S1474 $-4422(18) 30295-3$

2. Lee A, Gilbert RM (2016) Epidemiology of Parkinson disease. Neurol Clin NA 34:955-965. https://doi.org/10.1016/j. ncl.2016.06.012

3. Dorsey ER, Constantinescu R, Thompson JP et al (2007) Projected number of people with Parkinson disease in the most populous nations, 2005 through 2030. Neurology 68:384-386. https://doi. org/10.1212/01.wnl.0000247740.47667.03

4. Rao SS, Hofmann L, Shakil A (2006) Parkinson's disease: diagnosis and treatment. Am Fam Physician 74:2046-2054

5. Postuma RB, Berg D, Stern M et al (2015) MDS clinical diagnostic criteria for Parkinson's disease. Mov Disord 30:1591-1601. https ://doi.org/10.1002/mds.26424

6. Goetz CG, Tilley BC, Shaftman SR et al (2008) Movement disorder society-sponsored revision of the unified Parkinson's 
disease rating scale (MDS-UPDRS): scale presentation and clinimetric testing results. Mov Disord 23:2129-2170. https://doi. org/10.1002/mds.22340

7. Sánchez-Ferro Á, Elshehabi M, Godinho C et al (2016) New methods for the assessment of Parkinson's disease (2005 to 2015): a systematic review. Mov Disord 31:1283-1292. https://doi. org/10.1002/mds.26723

8. Sun Y, Yu XB (2016) Capacitive biopotential measurement for electrophysiological signal acquisition: a review. IEEE Sens J 16:2832-2853. https://doi.org/10.1109/JSEN.2016.2519392

9. Siegel JD, Rhinehart E, Jackson M, Chiarello L (2007) 2007 guideline for isolation precautions: preventing transmission of infectious agents in health care settings. Am J Infect Control 35:S65S164. https://doi.org/10.1016/j.ajic.2007.10.007

10. Braun A, Wichert R, Kuijper A, Fellner DW (2015) Capacitive proximity sensing in smart environments. J Ambient Intell Smart Environ 7:483-510. https://doi.org/10.3233/AIS-150324

11. Oliveira FHM, Zaruz TF, Vieira MF, Andrade AO (2019) t-SNE applied to discriminate healthy individuals from those with Parkinson's disease executing motor tasks detected by non-contact capacitive sensors. In: IFMBE proceedings, pp 421-425. https:// doi.org/10.1007/978-981-10-9035-6_78

12. Oliveira FHM, Zaruz TF, Andrade AO (2019) Use of non-contact capacitive sensors to detect hand gestures. In: Costa-Felix R, Machado JC, Alvarenga AV (eds) XXVI Brazilian congress on biomedical engineering. Springer, Singapore, pp 517-522. https:// doi.org/10.1007/978-981-13-2517-5_78

13. Prance $H$ (2011) Sensor developments for electrophysiological monitoring in healthcare. In: Gargiulo G, McEwan A (eds) Applied biomedical engineering. InTech, pp 265-286. https:// doi.org/10.5772/22958

14. González-Sánchez C, Fraile J-C, Pérez-Turiel J et al (2016) Capacitive sensing for non-invasive breathing and heart monitoring in non-restrained, non-sedated laboratory mice. Sensors 16:1052. https://doi.org/10.3390/s16071052

15. Pouryazdan A, Prance RJ, Prance H, Roggen D (2016) Wearable electric potential sensing. In: Proceedings of the 2016 ACM international joint conference on pervasive and ubiquitous computing adjunct - UbiComp'16. ACM Press, New York, USA, pp 846-850. https://doi.org/10.1145/2968219.2968286

16. Beardsmore-Rust S, Stiffell PB, Prance $\mathrm{H}$ et al (2010) Passive tracking of targets using electric field sensors. In: Carapezza EM (ed) Sensors, and Command, Control, Communications, and Intelligence (C3I) Technologies for Homeland Security and Homeland Defense IX. SPIE, Orlando, Florida, United States, $p$ 766622. https://doi.org/10.1117/12.849642

17. Plessey Semiconductors Ltd. Application Note \# 291554 Movement and gesture applications of EPIC sensors. 1-3

18. Winter B (2013) Linear models and linear mixed effects models in $\mathrm{R}$ with linguistic applications. arXiv:1308.5499. [http://arxiv .org/pdf/1308.5499.pdf]
19. Machado ARP, Zaidan HC, Paixão APS et al (2016) Feature visualization and classification for the discrimination between individuals with Parkinson's disease under levodopa and DBS treatments. Biomed Eng Online 15:169. https://doi.org/10.1186/ s12938-016-0290-y

20. Rabelo AG, Neves LP, Paixão APS et al (2017) Objective assessment of Bradykinesia estimated from the wrist extension in older adults and patients with Parkinson's disease. Ann Biomed Eng 45:2614-2625. https://doi.org/10.1007/s10439-017-1908-3

21. Andrade AO, Ferreira LCV, Rabelo AG et al (2017) Pelvic movement variability of healthy and unilateral hip joint involvement individuals. Biomed Signal Process Control 32:10-19. https:// doi.org/10.1016/j.bspc.2016.10.008

22. Andrade AO, Kyberd P, Nasuto SJ (2008) The application of the Hilbert spectrum to the analysis of electromyographic signals. Inf Sci 178:2176-2193. https://doi.org/10.1016/j.ins.2007.12.013

23. Elbaz A, Carcaillon L, Kab S, Moisan F (2016) Epidemiology of Parkinson's disease. Rev Neurol 172:14-26. https://doi. org/10.1016/j.neurol.2015.09.012

24. Patel S, Lorincz K, Hughes R et al (2009) Monitoring motor fluctuations in patients with Parkinson's disease using wearable sensors. IEEE Trans Inf Technol Biomed 13:864-873. https://doi. org/10.1109/TITB.2009.2033471

25. Roy SH, Cole BT, Gilmore LD et al (2013) High-resolution tracking of motor disorders in Parkinson's disease during unconstrained activity. Mov Disord 28:1080-1087. https://doi.org/10.1002/ mds. 25391

26. Chang R-S, Chiu J-H, Chen F-P et al (2011) A Parkinson's disease measurement system using laser lines and a CMOS image sensor. Sensors 11:1461-1475. https://doi.org/10.3390/s110201461

27. Beardsmore-Rust S, Watson P, Stiffell PB, et al (2009) Detecting electric field disturbances for passive through-wall movement and proximity sensing. In: Cullum BM, Porterfield DM (eds) Proc. of SPIE. $p$ 73130P

28. Aydin A, Stiffell PB, Prance RJ, Prance H (2010) A high sensitivity calibrated electric field meter based on the electric potential sensor. Meas Sci Technol 21:125901. https://doi. org/10.1088/0957-0233/21/12/125901

29. Spasojević S, Ilić TV, Stojković I et al (2017) Quantitative assessment of the arm/hand movements in parkinson's disease using a wireless armband device. Front Neurol. https://doi.org/10.3389/ fneur.2017.00388

Publisher's Note Springer Nature remains neutral with regard to jurisdictional claims in published maps and institutional affiliations. 